\title{
Computational Study on the Effect of Turbulence Intensity and Pulse Frequency in Soot Concentration in an Acetylene Diffusion Flame
}

\author{
Fernando Lopez-Parra and Ali Turan \\ School of Mechanical, Aerospace and Civil Engineering, \\ The University of Manchester, Po Box 88, \\ M60 1QD, Manchester, UK \\ F. Lopez-Parra@postgrad.umist.ac.uk
}

\begin{abstract}
A computational investigation of the effect of turbulence structure in the formation and depletion of soot in non-premixed acetylene turbulent diffusion flames is presented. Two separate modelling approaches are investigated: 1. Realizable k- $\varepsilon$ turbulence model combined with non-adiabatic strained laminar flamelets to solve the reaction mechanisms accounting for the effect of nonequilibrium species and, 2. Standard k- $\varepsilon$ turbulence model and Eddy-Break-Up -EBU-with volumetric global reaction and Eddy-Dissipation model for chemistry. In both cases the results obtained show that increments in the input Reynolds number yield lower concentrations of soot. It was also found that low frequency sinusoidal pulse in the fuel inlet velocity can contribute to further reduce the soot concentration in the flame. The soot and nuclei source codes were solved as post-processed scalars and considered to be "passive" species.
\end{abstract}

\section{Introduction}

The main objective of the present work is the creation of a mathematical subroutine that would reproduce a soot model based on the eddy dissipation concept of Magnus$\operatorname{sen}^{1-5}$ and that can be implemented into a commercial solver. The performance of the sub-routine is tested with a 2-dimensional axi-symmetric diffusion acetylene flame. Over the last few decades, there has been a growing interest in the modeling of particulates from combustion systems. Although the mechanisms of soot formation are not completely understood, the behavior of this carcinogenic ${ }^{6,7}$ specie has been computationally reproduced in the past with an acceptable degree of accuracy ${ }^{1-5,8-10}$. There are two differentiated stages in which soot forms ${ }^{11}$ : the inception of the particles for which soot will ultimately form -nucleation- and the subsequent growth of these particles. The growth of the carbonaceous particles takes place in two different stages, referred to as the agglomeration and the surface growth. Soot originates from the $\mathrm{C}_{2}$ radicals that form as a consequence of the break down -pyrolysis- of the fuel molecule. In turbulent chemical reactions, there is a strong influence of the flow parameters in the performance of the combustion. As a result, an inhomogeneous structure of the appearance of reacting species will develop. In these situations, the molecular mixing of the fuel and oxidant, which is highly intermittent, takes inside the fine 
structures, which occupy a fraction of the total volume of the domain. These are believed to be three-dimensional vortex tubes of very small dimensions in one or two directions, but not in the other one. These are vortex are of the same characteristic dimensions as the Kolmogorov structures ${ }^{13}$. These regions for the last link in the turbulence energy transfer cascade, and in modeling environments, it is assumed that fuel and oxidant are perfectly mixed. In general, high turbulent flows of high Reynolds number would present a spectrum of eddies of different sizes. These eddies transfer mechanical energy to their immediate neighbors and the interaction between the larger and the smaller eddies represents the main source of production of turbulence kinetic energy. On the other hand, the dissipation of kinetic energy into heat, due to the work done by molecular forces on the eddies, takes place in the smallest eddies.

The mass fraction occupied by the fine structures is given by

$$
\gamma^{*}=9.7 \cdot\left(\frac{v \cdot \varepsilon}{k^{2}}\right)^{0.75}
$$

where $\mathrm{k}$ is the turbulent kinetic energy -TKE-, where $v$ is the kinematic viscosity and $\varepsilon$ is the turbulence dissipation rate -TDR .

The mass transfer per unit mass and unit time between the fine structures and the surrounding fluid is expressed by

$$
\dot{m}=23.6\left(\frac{v \cdot \varepsilon}{k^{2}}\right)^{0.25} \frac{\varepsilon}{k}
$$

Due to the reaction taking place within the fine structures, these will have a higher temperature with respect to the local mean temperature. The increment of temperature, $\Delta \mathrm{T}$, is computed as

$$
\Delta T=\frac{\Delta H_{R} \cdot c_{\text {min }}}{\rho \cdot c_{p}}
$$

where $\Delta \mathrm{H}_{\mathrm{R}}$ is the heat of reaction of the fuel, $\rho$ is the density, $\mathrm{c}_{\mathrm{p}}$ is the local specific heat capacity of the mixture and $\mathrm{c}_{\min }$ is the minimum of $\mathrm{c}_{\mathrm{fu}}$ and $\mathrm{c}_{\mathrm{o} 2} / \mathrm{r}_{\mathrm{fu}}$, where $\mathrm{c}_{\mathrm{fu}}$ and $\mathrm{c}_{\mathrm{o} 2}$ are the gravimetric concentrations of fuel and oxygen respectively and $\mathrm{r}_{\mathrm{fu}}$ is the gravimetric oxygen requirement to burn $1 \mathrm{~kg}$ of fuel.

In the Magnussen model, the fine structures and the surrounding fluid are assumed to be in local equilibrium and the concentration of species in the fine structures and the surrounding fluid are related to the local mean concentrations by

$$
\frac{c_{i}}{\rho}=\frac{c_{i}^{*}}{\rho^{*}} \gamma^{*} \chi+\frac{c_{i}^{o}}{\rho^{o}}\left(1-\gamma^{*} \chi\right)
$$

where $\chi$ is a local variable that accounts for the fraction of fine structures that are actually heated enough to react and $\mathrm{c}$ is the concentration of species. 
After defining all the preliminary and more relevant variables in the model, the mean nuclei and soot formation rates are expressed as follows

$$
\begin{aligned}
& R_{n, f}=\left(n_{o}^{*} \frac{\gamma^{*} \chi}{\rho^{*}}+n_{o}^{o} \frac{1-\gamma^{*} \chi}{\rho^{o}}+g_{o} n^{*} \frac{\gamma^{*} \chi}{\rho^{*}}\left(N^{o}-N^{*}\right)\right) \rho+(f-g) \cdot Y_{n и с} \cdot \rho-g_{0} \rho \mathrm{N}^{o} Y_{n и с} \\
& R_{s, f}=m_{p}\left(a \cdot Y_{n u c} \cdot \rho+b N^{*} \frac{\gamma^{*} \chi}{\rho^{*}} \rho\left(n^{o}-n^{*}\right)\right)-\rho b n^{o} Y_{\text {soo }}
\end{aligned}
$$

where $Y_{\text {nuc }}$ and $Y_{\text {soot }}$ are the mass fractions of nuclei and soot, $\mathrm{N}$ is the soot particle concentration and ( $\mathrm{f}-\mathrm{g}$ ) is the nuclei branching-termination coefficient and $\mathrm{a}, \mathrm{b}$ and $\mathrm{g}_{\mathrm{o}}$ are model constants.

The mean rates of nuclei and soot oxidation are comparable to the rate of fuel combustion.

$$
R_{\text {pollut }, c}=\dot{m} \cdot \chi \cdot c_{\min } \frac{Y_{p o l l u t}}{Y_{f u}}
$$

The net rates of nuclei and soot formation were computed by subtracting the rate of combustion from the rate of formation. The resulting equation was used as the source term for soot and nuclei in the user-defined function written for FLUENT. One of the major challenges presented in was the appropriate selection of a linearization term. Source terms in FLUENT are written as a two-part variable, with well differentiated implicit and explicit sides, e.g. $S_{\phi}=A+B \phi$, where $\phi$ would be the dependent variable and $\mathrm{A}$ and $\mathrm{B} \phi$ would be the explicit and implicit parts respectively. FLUENT automatically determines whether the given expression for B enhances the stability and if this is not the case, the source term is handled explicitly. In current work, algebraic manipulation of the expressions given above yielded the separate explicit and implicit terms required for the simulation.

This soot model also relies in the correct specification of the model constants. Whereas some of these are rather universal, the soot particle diameter, $d_{p}$, and the preexponential constant for nucleation, $\mathrm{a}_{\mathrm{o}}$ need to be adjusted for different flow conditions. In the present work, the two following expressions have been used, combined with the experimental measurements of Magnussen ${ }^{5}$ to determine these parameters for each case:

$$
\begin{aligned}
& \dot{m} \cdot a_{o}=\text { constant } \\
& d_{p}=\frac{D \cdot c_{f u, o}^{1.6}}{U_{o}^{0.6}}\left(\frac{\rho_{f l}}{\rho_{o}}\right) \frac{1}{v_{f l}^{0.2}} \cdot \text { constant }
\end{aligned}
$$

where sub-index $o$ and $f l$ refer to inlet and flame conditions respectively.

The constants employed in the turbulence model are given in Table I, and the turbulence viscosity was user-defined as

$$
\mu_{t}=\frac{\rho \cdot C_{D} \cdot k^{2}}{\varepsilon}
$$


Table 1. Turbulence model constants

\begin{tabular}{|c|c|c|c|c|c|c|c|c|c|}
\hline $\mathrm{C}_{\mathrm{D}}$ & $\mathrm{C}_{1}$ & $\mathrm{C}_{2}$ & $\sigma_{\mathrm{h}}$ & $\sigma_{\mathrm{k}}$ & $\sigma_{\varepsilon}$ & $\sigma_{\mathrm{fu}}$ & $\sigma_{\mathrm{f}}$ & $\sigma_{\mathrm{s}}$ & $\sigma_{\text {nuc }}$ \\
\hline 0.09 & 1.44 & 1.79 & 0.7 & 1.0 & 1.3 & 0.7 & 0.7 & 0.7 & 0.7 \\
\hline
\end{tabular}

The basic transport equations solved in these simulations can be written in a general for as follows

$$
\frac{\partial}{\partial x}(\bar{\rho} \bar{U})+\frac{1}{y} \frac{\partial}{\partial y}(y \bar{\rho} \bar{V})=0, \text { for mass }
$$

and for momentum and scalar quantities:

$$
\bar{\rho} \bar{U} \frac{\partial \phi}{\partial x}+\bar{\rho} \bar{V} \frac{\partial \phi}{\partial y}=\frac{1}{y} \frac{\partial}{\partial y}\left(y \frac{\mu_{t}}{\sigma_{\phi}} \frac{\partial \phi}{\partial y}\right)+R_{\phi}
$$

All transport equations were solved using a second order upwind discretization scheme, except the turbulence dissipation rate. In the case of soot and nuclei, QUICK discretization scheme was employed. The convergence criteria were $1 \mathrm{E}-3$ for most parameters, apart from the energy and soot, for which it was set to $1 \mathrm{E}-6$ and $1 \mathrm{E}-5$ respectively. In reacting flows, the fact that the flow has reached convergence does not necessarily imply that an equilibrium state has been reached. Thus, the concentrations of some product species were monitored at points near the exit of the combustor.

\section{Procedure}

There are three wee differentiated areas in the present work: the implementation of the Magnussen model using both flamelet and EBU chemistry models, the analysis of the effect of Reynolds number in the final soot concentration and the effect of sinusoidal fuel pulses in the in also the soot concentration.

Nowadays there is a varied choice for turbulence and combustion models, and it cannot be said that one outperforms the others in all flow conditions. It is normally good practice to decide the models to employ by solving a flame or combustion environment that closely resembles the one subject to investigation. In this case, a turbulent piloted diffusion flame $e^{13-15}$-referred to as flame D- was reproduced in order to evaluate the performance of the turbulence model and the combustion model. In order to improve the predictions of the spreading of the diffusion jet, realizable $\mathrm{k}-\varepsilon$ was chosen to model the momentum transport equations. Based on the good agreement of the reproduced flame D in FLUENT with the results obtained with conditional moment closure ${ }^{13,14}$ and the experimental data ${ }^{15}$, this methodology was assumed to be suited for the simulation of 2-dimensional axi-symmetric diffusion flames. Despite this work being based on the findings of Magnussen, the boundary conditions were modified due to a lack of boundary condition information such as the shape of the issuing nozzle or the TKE and TDR -which can be critical for combustion simulation-, the grid employed, or any discussion as to how the flame can be stable at the given fuel inlet velocity. Therefore, the fuel inlet velocities were reduced with 
respect to those employed in references ${ }^{1-5}$. In the present study, jet Reynolds numbers of $3300,16500,20000$ and $25000-\mathrm{U}_{\mathrm{jet}}=10,50,60$ and $75 \mathrm{~m} / \mathrm{s}$ respectively- were tested using the flamelet model and Reynolds numbers of 8300, 11500 and 16500 for when the EBU was employed. A sinusoidal fuel inlet delivery was also analysed, with frequencies 50, 100 and $200 \mathrm{~Hz}$ and amplitude of $25 \mathrm{~m} / \mathrm{s}$. The time steps were adjusted in each case to ensure 20 divisions in each complete cycle and $2^{\text {nd }}$ Order Implicit was employed for the unsteady discretization.

\section{Results}

The first part of the study was the simulation of flame D using the flamelet model in order to see how it compares to the CHEMKIN mechanisms used in the literature. Figure 1 depicts the values of mixture fraction at the $\mathrm{x} / \mathrm{d}=15$ station of the piloted flame D. This result is in close agreement with the experimental data ${ }^{15}$ and predictions made using detailed chemical mechanism ${ }^{13,14}$ Likewise, the temperature estimation and the main species mass fractions -figure 3- are within reasonable agreement with experiments. The axial velocity was also compared at the different stations, again showing good agreement with the sources of data. As a result of this study, it was concluded that the flamelet model and the selected realizable k- $\varepsilon$ turbulence model were suited to the simulation of turbulent diffusion flames, and therefore were also used to simulate the acetylene flame. The flame D simulation was also employed as an initial step into the grid-independence study, based on both temperatures and species concentrations mainly, and is later applied to the acetylene investigation.

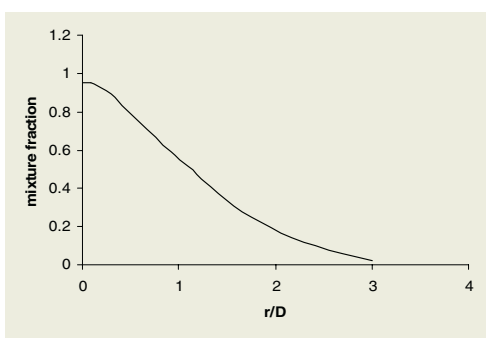

Fig. 1. Flame D, mixture fraction

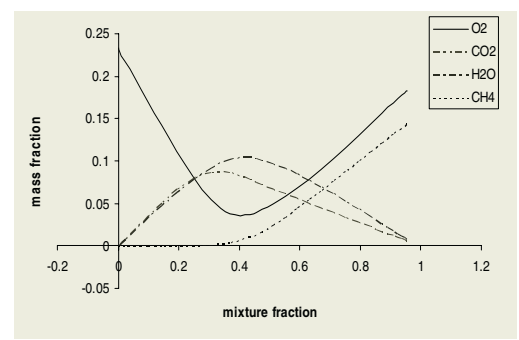

Fig. 2. Flame D, species mass fraction

In the case of the acetylene combustion, the geometry differed slightly from what it can be inferred from the literature. The major differences are that in the present case a converging nozzle is not employed and the issuing jet is not placed in the centre of a circular plate, and instead is placed in an unconfined environment. Figure 3 depicts the turbulence dissipation rate near the jet exit, where a large difference in the dissipation values is observed. These variations of turbulence dissipation will have an effect on the soot and nuclei formation rates, but this effect is more clearly seen when combined with the turbulence kinetic energy in order to monitor the turbulence time scale, as depicted in figure 4. In this figure a large reduction in the time scale is observed between the low Re and the other higher Re conditions. In can also be appreciated that the decrease of the eddy lifetime is also non-linear, since the decrease between 
$\mathrm{Re}=16500$ and $\mathrm{Re}=20000$ is larger than that between $\mathrm{Re}=20000$ and $\mathrm{Re}=25000$. These results imply that the residence time allowed for soot to form and growth in the higher Reynolds number conditions is reduced compared to those lower Reynolds. The effect of the turbulence intensity on the formation and combustion of soot is depicted in figure 5. It can be seen that for lower Re, the peak soot concentration is closer to the nozzle that for the higher Re. However, one other feature of this graph is the fact that the Reynolds number does not have a strong effect on the location of the peak soot concentration for higher Re. On the other hand, it does have a strong impact on the maximum soot mass fraction, which reduces as Re increases. Consequently, it can be said that the input boundary conditions do have a significant influence on the soot concentration in turbulent flames. However, it can also be seen that there is a deviation of the peak of the soot concentration towards the exit of the combustor. These differences in are thought to be due to an inappropriate linearization of the scalar transport equations and are currently under study.

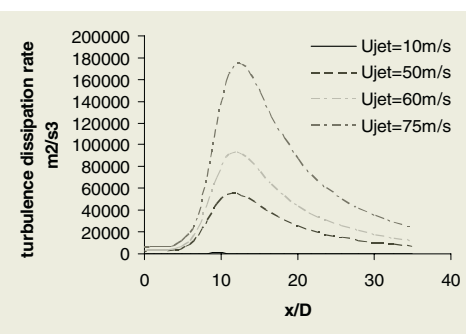

Fig. 3. Acetylene, turbulence dissipation rate

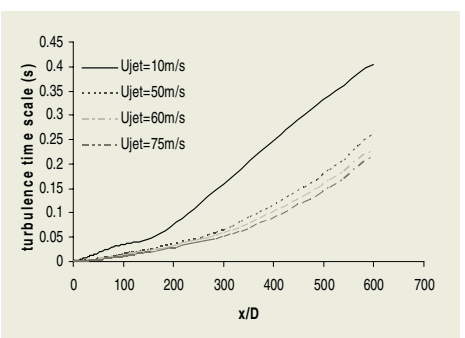

Fig. 4. Acetylene, turbulence time scale

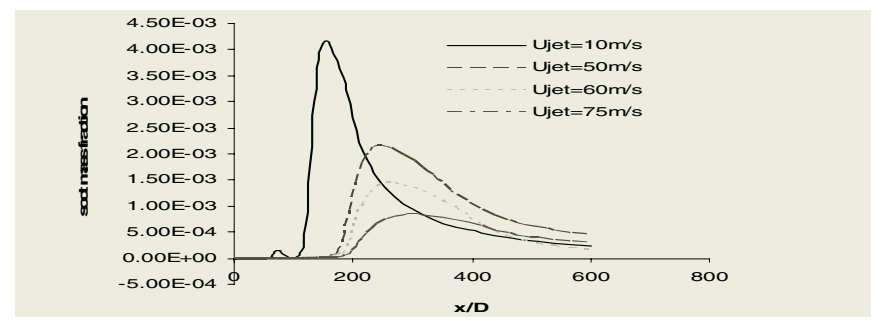

Fig. 5. Acetylene, soot mass fraction at different Re

A similar effect of the Reynold number on the final concentration of soot was observed when the EBU was employed. Comparing the results directly with those obtained with the flamelet model, the EBU presents the peak soot concentrations in the areas of the flame where it could be expected, unlike the flamelet model. This is probably due to the incompatibility of the flamelet model, which assumes that fuel and oxidizer cannot co-exist, unless the strain rate extinguishes the flame, and Magnussen soot model assumes that the fine structures and the surrounding flow are in equilibrium, which seems to be contradictory with the flamelet. Figure 6 depicts the soot concentration along the symmetry axis for the three fuel inlet velocities studied. 
This trend also seen along the radial lines, like the example take from a vertical station $0.3 \mathrm{~m}$ downstream the jet exit.

These results indicate that increased levels of turbulence can, indeed, reduce the soot emissions from a combustion reaction. One other factor that can enhance the depletion of soot particles is the entrainment of oxygen into the core of the diffusion flame, i.e. the fuel rich area. This could be achieved by pulsing the fuel, thus lengthening and twisting the shear layer through which air and fuel ultimately mix. Figures 8, 9 and 10 depict a comparison between the root-mean-square -rms- of the soot concentrations for the three frequencies investigated and the steady state concentration along the symmetry axis and two vertical stations located 0.2 and $0.3 \mathrm{~m}$ downstream the nozzle exit. The pulsed-fuel cases were run for a total flow time of about $2.7 \mathrm{sec}$ onds to allow the pulse to stabilize. Thus we assumed that the quantities seen in one pulse would be repeated in the next one.

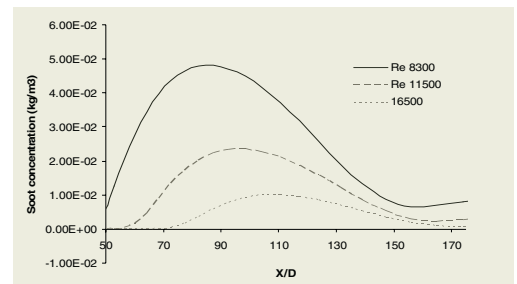

Fig. 6. Axial Soot concentration

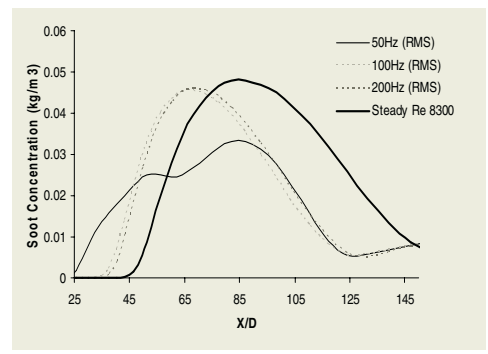

Fig. 8. Soot rms along axis

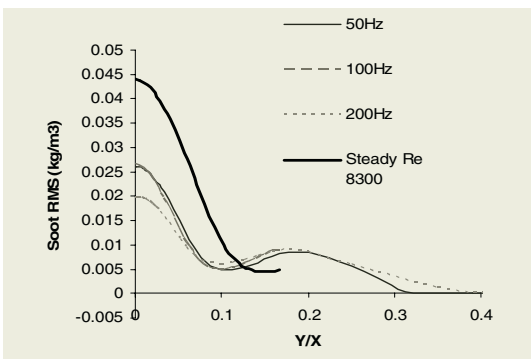

Fig. 10. Soot rms at $X=0.3 m$

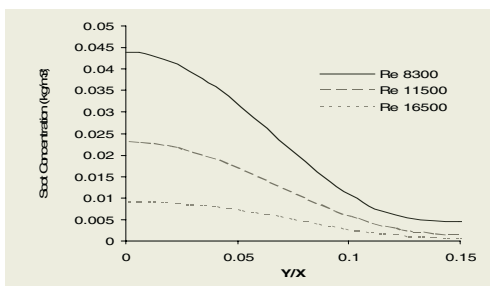

Fig. 7. Radial soot concentration at $X=0.3 \mathrm{~m}$

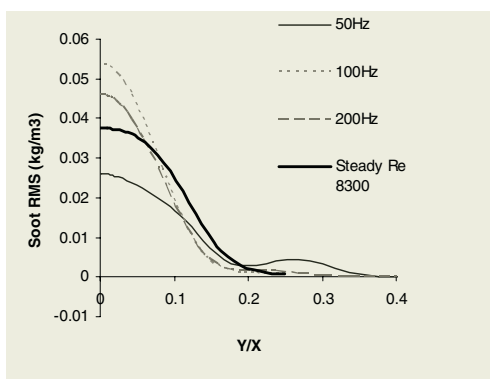

Fig. 9. Soot $r m s$ at $X=0.2 m$

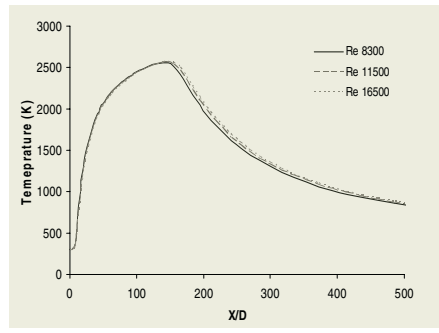

Fig. 11. Axial temperature, steady state 
Figure 11 depicts the temperature predicted during the steady state simulations at different Reynolds numbers. Because the temperature is very similar in each case, the reduction in soot concentration must come from the difference in the turbulent kinetic energy and dissipation, as seen in the flamelet case. It is not surprising to find a higher concentration of soot with the pulsed fuel respect to the steady state in Figure 9 because, as can be appreciated in Figure 10, the peak of soot concentration is moves towards the jet exit when the fuel is pulsed. Thus, a more realistic reading of the effect of pulsing in the soot concentration can be made from Figure 10.

\section{Conclusions}

A soot model with turbulence interaction has been satisfactorily implemented onto a commercial code. At present work is being done to treat soot as"active" specie.

The flamelet model has proved to produce very good thermodynamic solutions, but seems to be incompatible with the soot model.

Both flamelet and EBU simulations have shown a decreasing concentration of soot with increasing flow Reynolds number, whilst temperature distribution has remained fairly unchanged.

Pulsing fuel has shown a decrease in the final concentration of soot based on stabilized pulsed flames and compared to a steady velocity equal to the amplitude of the pulse. Should it be compared to the rms of the inlet velocity, these results would be even larger.

\section{References}

1. Magnussen, B.F. On the structure of turbulence and generalized eddy dissipation conceptfor chemical reaction in turbulent flow. $19^{\text {th }}$ AIAA Science Meeting, 1981.

2. Magnussen, B.F. Modelling of NOx and soot formation by the eddy dissipation concept. International Flame Foundation First Topic Oriented Technical Meeting, 1989.

3. Magnussen, B.F. $15^{\text {th }}$ Symposium (Int) on Combustion. The Combustion Institute, Pittsburgh, 1974.

4. Magnussen, B.F., Hjertager, B.H. $16^{\text {th }}$ Symposium (Int) on Combustion. The Combustion Institute, Pittsburgh, 1976.

5. Magnussen, B.F., Hjertager, B.H, Olsen, J.G, Bhaduri, D. $17^{\text {th }}$ Symposium (Int) on Combustion. The Combustion Institute, Pittsburgh, 1979.

6. Thilly, W.G. Soot Combustion Systems and its Toxic Properties, pp1-12, Plenum Press, New York, 1983.

7. Boyland, E. Soot Combustion Systems and its Toxic Properties, pp13-24, Plenum Press, New York, 1983.

8. Tesner, P.A., Snegiriova, T.D., Knorre, V.G. Combustion and Flame, 17, pp253-260, 1971.

9. Tesner, P.A., Tsygankova, E.I., Guilazetdinov, L.P., Zuyev, V.P., Loshakova, G.V. Combustion and Flame, 17, pp 279-285, 1971.

10. Srivatsa, S.K. NASA-Lewis Research Center, NAS3-22542, NASA CR-167930, Garrett 214309, 1982. 
11. Prado, G., Lahaye, J., Haynes, B.S. Soot Combustion Systems and its Toxic Properties, pp145-162, Plenum Press, New York, 1983.

12. Kolmogorov, A.N., Journal of Fluid Mechanics, 13, 82, 1962.

13. Fairweather, M., Woolley, R.M., Combustion and Flame, 138, pp3-19, 2004.

14. Fairweather, M., Woolley, R.M. Combustion and Flame, 133, pp393, 2003.

15. Information available at http://www.ca.sandia.gov/TNF 